\title{
Plant responses to multiple antagonists are mediated by order of attack and phytohormone crosstalk
}

\author{
Saumik Basu ${ }^{1}$, Robert Clark ${ }^{1}$, Sayanta Bera ${ }^{2}$, Clare Cateel$^{2}$, and David Crowder ${ }^{1}$ \\ ${ }^{1}$ Washington State University \\ ${ }^{2}$ Cornell University
}

April 7, 2021

\begin{abstract}
Plants are often attacked by multiple antagonists, and traits of the attacking organisms, and their order of arrival onto hosts, may affect plant defenses. However, few studies have assessed how multiple antagonists, and varying attack order, affect plant defense or nutrition. To address this, we assessed defensive and nutritional responses of Pisum sativum plants after attack by a vector herbivore (Acrythosiphon pisum), a non-vector herbivore (Sitona lineatus), and a pathogen (Pea enation mosaic virus, PEMV). We show PEMV-infectious A. pisum induced several pathogen-specific plant defense signals, but these defenses were inhibited when $S$. lineatus was present in peas infected with PEMV. In contrast, feeding by $S$. lineatus induced anti-herbivore defense signals, but these defenses were enhanced by PEMV. Sitona lineatus also increased abundance of plant amino acids, but only when they attacked after PEMV-infectious A. pisum. Our results suggest that diverse communities of biotic antagonists alter defense and nutritional traits of plants through complex pathways that depend on the identity of attackers and their order of arrival onto hosts. Moreover, we show interactions among a group of biotic stressors can vary along a spectrum from antagonism to enhancement/synergism based on the identity and order of attackers, and these interactions are mediated by a multitude of phytohormone pathways.
\end{abstract}

\section{INTRODUCTION}

Plant hosts have defenses to counter attacks from antagonists such as herbivores and pathogens (Pandey, Ramegowda, \& Senthil-Kumar, 2015; Miller, Costa Alves, \& Van Sluys, 2017). For example, the jasmonic acid pathway often regulates plant defenses against herbivores, while the salicylic acid pathway often regulates defenses against pathogens (Koornneef \& Pieterse, 2008; Thaler, Humphrey, \& Whiteman, 2012). While many studies have assessed plant responses to particular antagonists, plants are often challenged by many stressors concurrently, and plant defenses can depend on the order in which antagonists arrive on plants (Thaler et al., 2012; Nejat \& Mantri, 2017). For example, herbivores often limit plant defenses against pathogens when they arrive first on plants, but herbivores often have few impacts on plant defenses against pathogens when they arrive after pathogens on plants (Okada, Abe, \& Arimura, 2015; Lin et al. , 2019). In other contexts, certain organisms 'prime' pathways, promoting defense against subsequent organisms activating the same pathway, such that attack order may not matter (Mauch-Mani, Baccelli, Luna, \& Flors, 2017; Ramírez-Carrasco, Martínez-Aguilar, \& Alvarez-Venegas, 2017).

Biotic stressors may also alter the nutritional quality of plants by regulating amino acid metabolism (Casteel et al., 2014; Zhou, Lou, Tzin, \& Jander, 2015), which can alter the feeding behavior and nutrient uptake by subsequent herbivores (Behmer, 2009; Zhu, Poelman, \& Dicke, 2014). For example, the composition of free amino acids constitutively changes in leaves of soybean plants in response to soybean aphids (Chiozza, O'Neal, \& MacIntosh, 2010). Tomato yellow leaf curl virus also alters the nutritional quality of tomato plants by affecting free amino acid levels in phloem, which alters the amino acid composition of whitefly (Bemisia 
tabaci ) honeydew (Guo et al., 2019). However, few studies have explored how the diversity and identity of attacking organisms, and variation in the order of attack, affect nutritional traits of plants.

While there has been considerable research on the jasmonic and salicylic acid pathways, to understand complexities of plant defense it is necessary to assess how biotic antagonists mediate other signaling pathways (e.g., Lacerda, Vasconcelos, Pelegrini, \& Grossi de Sa, 2014; Suzuki, 2016). Moreover, it is key to assess how changes in plant defense correlate with plant nutrients. For example, plants in low-nitrogen soil often adopt carbon-based defenses, while plants grown with fertilizer often accumulate more nitrogenous toxins (Cipollini, Walters, \& Voelckel, 2017). Nitrogen in plants may also affect both pathogens and herbivores through synthesis of defensive metabolite, nitric oxide, and by nitrogen mobilization (War et al., 2012; Mur, Simpson, Kumari, Gupta, \& Gupta, 2017). However, few studies have correlated effects of multiple biotic stressors on both plant chemical signaling and nutritional properties (Petek et al., 2014; Su et al., 2016).

We addressed these knowledge gaps by assessing the response of Pisum sativum plants to attack from a piercing-sucking vector herbivore, the pea aphid (Acrythosiphon pisum ), a chewing non-vector herbivore, the pea leaf weevil (Sitona lineatus), and an aphid-borne pathogen, Pea-enation mosaic virus (PEMV). These organisms co-occur in ecosystems of eastern Washington and northern Idaho, USA, and interactions between them can affect plant traits and signaling pathways affecting insects and pathogens (Chisholm, Eigenbrode, Clark, Basu, \& Crowder, 2019; Bera, Blundell, Liang, Crowder, \& Casteel, 2020). However, the order in which herbivores and pathogens arrive on hosts, which varies across sites (Chisholm et al., 2019), may impact plant traits and defenses. To address this, we varied the diversity, identity, and order of attack among this community of biotic antagonists and assessed resulting changes in gene expression and phytohormones related to plant defense and nutrition. Our study revealed how plant responses to diverse stressors can mediate complex species interactions within a pathosystem.

\section{MATERIALS AND METHODS}

\subsection{Study system}

The Palouse region of eastern Washington and northern Idaho, USA, is home to many legumes including $P$. sativum (Black et al., 1998). In P. sativum fields, S. lineatus, a chewing herbivore, co-occurs with A. pisum , a phloem-feeding herbivore that can transmit pathogens such as PEMV (Chisholm et al., 2019). PEMV is one of several viruses that infects $P$. sativum, and this pathogen is obligately transmitted by aphids in a persistent manner (Chisholm et al., 2019).

Sitona lineatus adults overwinter outside of $P$. sativumfields and migrate into fields in late spring before A. pisumarrive (Cárcamo et al., 2018). After S. lineatus eggs hatch, larvae burrow into the soil to feed and pupate before emerging as adults in the summer (Cárcamo et al., 2018); these second-generation adults often occur on plants under attack from A. pisum and PEMV (Chisholm et al., 2019). Thus, S. lineatus attacks plants in the field both before and after A. pisum and PEMV. However, it is unknown if responses of $P$. sativum differ based on the number of stressors, and their order of attack. Moreover, molecular mechanisms that mediate interactions among these stressors are largely unknown (Chisholm et al., 2019; Bera et al., 2020).

To address these questions, we conducted greenhouse assays to assess interactions between $S$. lineatus , A. pisum, and PEMV onP. sativum plants, and molecular mechanisms affecting these interactions. Firstgeneration adult $S$. lineatus for experiments were collected from commercial $P$. sativum fields, or wild patches of Vicia villosa, immediately prior to experiments. Colonies of infectious A pisum with PEMV, and uninfectious A. pisum, were started from Palouse field-collected individuals (Chisholm et al., 2019) and were maintained on P. sativum plants in a greenhouse $\left(21-24^{\circ} \mathrm{C}\right.$ during day cycle, $16-18^{\circ} \mathrm{C}$ during dark cycle, 16:8 h light:dark).

\subsection{Experimental design}

We conducted a $3 \times 2$ greenhouse $\left(21-24^{\circ} \mathrm{C}\right.$ day cycle, $16-18^{\circ} \mathrm{C}$ dark cycle, $16: 8 \mathrm{~h}$ light:dark $)$ experiment that varied S. lineatus , A. pisum, and PEMV (Fig. 1). There were three S. lineatustreatments: (i) control: no 
adults prior to A. pisum treatments (none), (ii) two adults that fed for $48 \mathrm{~h}$ prior to A. pisumtreatments (first), and (iii) two adults that fed for $48 \mathrm{~h}$ after A. pisum treatments (second). The two A. pisum treatments were: (i) sham: 10 5-d old uninfectious adults that fed for $48 \mathrm{~h}$ and (ii) PEMV: 10 5-d old PEMV-infectious adults that fed for $48 \mathrm{~h}$. For treatments with $S$. lineatus first, they were removed by hand prior to A. pisum treatments; for treatments with $A$. pisumfirst, they were removed by aspirator prior to $S$. lineatustreatments. Treatments were conducted on individual $P$. sativumplants in mesh 'bug dorms' $(0.6 \times 0.6 \times 0.6 \mathrm{~m})$, with six replicates randomly assigned to each treatment in a factorial design $(3 \mathrm{~S}$. lineatus treatments $\times 2$ A. pisum treatments). After insects were removed, plants were allowed to develop for $7 \mathrm{~d}$ before we harvested tissue to assess viral titer, gene expression, and nutrients. Tissue samples from the whole aboveground portion plants were collected and flash frozen in liquid nitrogen and stored in a $-80^{\circ} \mathrm{C}$ freezer until processing. Viral titer samples confirmed that $100 \%$ of plants in the PEMV treatments became infected over the course of the experiment.

\subsection{Analysis of plant defense and biosynthetic genes}

Plant tissue was processed using liquid nitrogen in sterilized mortars and pestles. Powdered tissue samples (50 to $100 \mathrm{mg}$ ) were used for RNA extraction with Promega SV total RNA isolation kits (Promega, Madison, WI). The quantity and quality of RNA was estimated on a NanoDrop1000 and agarose gel electrophoresis, respectively and $1 \mu \mathrm{g}$ of total RNA from each sample was used for cDNA synthesis (Bio-Rad iScript cDNA Synthesis kits). Gene specific primers (Table S3) for qRT-PCR were designed using the IDT Primer Quest Tool. Each qRT-PCR reaction $(10 \mu \mathrm{l})$ was set up containing $3 \mu \mathrm{l}$ of ddH2O, $5 \mu \mathrm{l}$ of iTaq Univer SYBR Green Supermix (Bio-Rad), $1 \mu \mathrm{l}$ of specific primer mix (forward and reverse [concentration $10 \mu \mathrm{M}]$ ), and $1 \mu \mathrm{l}$ of diluted (1: 25) cDNA template. Reactions were set up in triplicates for each sample and ran on a CFX96 qRT-PCR machine (Biorad). The qRT-PCR program included an initial denaturation for 3 min at $95^{\circ} \mathrm{C}$, followed by 40 denaturation cycles for $15 \mathrm{~s}$ at $95^{\circ} \mathrm{C}$, annealing for $30 \mathrm{~s}$ at $60^{\circ} \mathrm{C}$, and extension for $30 \mathrm{~s}$ at $72^{\circ} \mathrm{C}$. For melting curve analysis, a dissociation step cycle $\left(55^{\circ} \mathrm{C}\right.$ for $10 \mathrm{~s}$ and then $0.5^{\circ} \mathrm{C}$ for $10 \mathrm{~s}$ until $\left.95^{\circ} \mathrm{C}\right)$ was added. The comparative $2^{-\mathrm{Ct}}$ method (Livak \& Schmittgen, 2001; Kozera \& Rapacz, 2013) was used to calculate the relative expression level of each gene, with $\beta$-tubulin as an endogenous control.

We assessed expression of seven genes associated with defense in peas. Gene sequences were obtained using accession numbers (available genes) or using Pea Marker Database (Kulaeva et al., 2017) and blast searching through the reference genome (Kreplak et al., 2019). Four genes were associated with plant hormone biosynthesis: (i) Isochorismate synthase1 (ICS1 ) (salicylic acid), (ii) Lipoxygenase 2(LOX2) (jasmonic acid), (iii) Aldehyde oxidase 3(AO3 ) (abscisic acid), and (iv) Gibberellin 2-oxidase(GA2ox ) (gibberellic acid). ICS1 converts chorismate to isochorismate, a precursor of salicylic acid biosynthesis (Seguel et al., 2018), while LOX2 is a precursor to jasmonic acid biosynthesis (Wasternack \& Hause, 2013). AO3 catalyzes abscisic acid biosynthesis by oxidizing abscisic aldehyde, and GA2OX catalyzes bioactive giberrelic acids or their immediate precursors to inactive forms (Zdunek-Zastocka \& Sobczak, 2013; Serova, Tsyganova, Tikhonovich, \& Tsyganov, 2019; He et al., 2019). All of these gene transcripts can affect plant defense and plant-microbe interactions (Lee et al., 2012; Yergaliyev et al., 2016).

The three additional genes examined were associated with defense response transcripts that occur downstream from hormone induction. One of these genes, Pathogenesis-related protein 1 (PR1) affects systemic acquired resistance-mediated defense signaling and occurs downstream in the salicylic acid pathway (Fondevilla, Küster, Krajinski, Cubero, \& Rubiales, 2011; Miranda et al., 2017). The second defense response transcript was an antimicrobial defensin peptide called Disease resistance response gene (DRR230), which has been reported to provide resistance in peas against various pathogens (Lacerda et al., 2014; Selim, Sanssené, Rossard, \& Courtois, 2017). The third defense response transcript assessed was Lectin (PsLectin) . Plant lectins are a group of carbohydrate binding proteins, and Lectin genes can be induced by salicylic acid, jasmonic acid, and herbivores to stimulate phytoalexin and pistatin production in peas (Fondevilla et al., 2011; Armijo et al., 2013; Macedo, Oliveira, \& Oliveira, 2015).

2.4 Measurement of plant phytohormones 
Plant tissue samples were assessed for three phytohormones: jasmonic acid, salicylic acid, and abscisic acid following procedures of Patton, Bak, Sayre, Heck, \& Casteel (2019). Briefly, tissue samples were first flash frozen in liquid nitrogen before being lyophilized and weighed. Hormones were extracted in isopropanol: $\mathrm{H}_{2} \mathrm{O}: \mathrm{HCL}_{1 \mathrm{MOL}}(2: 1: 0.005)$ with $100 \mu \mathrm{l}$ of internal standard solution (1000 pg of each). Samples were evaporated to dryness, resuspended in $100 \mu \mathrm{l}$ of $\mathrm{MeOH}$, filtered, and $10 \mu \mathrm{l}$ of each sample was injected into an Agilent Technologies 6420 triple quad liquid chromatography-tandem mass spectrometry instrument (Agilent, Santa Clara, CA). A Zorbax Extend-C18 column $3.0 \times 150 \mathrm{~mm}$ (Agilent, Santa Clara, CA) was used with $0.1 \%$ formic acid in water $(\mathrm{A})$ and $0.1 \%(\mathrm{v} / \mathrm{v})$ formic acid in acetonitrile $(\mathrm{B})$ at a flow rate of 600 $\mathrm{mL} \mathrm{min}^{-1}$. The gradient used was 0-1 min, $20 \% \mathrm{~B} ; 1-10 \mathrm{~min}$, linear gradient to $100 \% \mathrm{~B} ; 10-13 \mathrm{~min}, 100 \%$ A. Retention times were: jasmonic acid (D5) standard (5.740 min), jasmonic acid (5.744 min), salicylic acid D4 standard (4.677 min), salicylic acid (4.720 min).

\subsection{Analysis of plant nutritional components}

For amino acid analysis, leaf tissue was lyophilized, weighed, and extracted with 20mM of HCL (Patton et al., 2019). Derivation was done using AccQTag reagents following the manufacturer's instructions (Waters, Shinagawa-ku, Tokyo), and derivatised samples $(10 \mu \mathrm{l})$ were then injected. Ground tissue was extracted with $100 \mu \mathrm{l}$ of $20 \mathrm{mM} \mathrm{HCl}$, centrifuged, and the supernatant was saved. Amino acids were derivatized using AccQ-Fluor reagent kits (Waters, Milford, MA), with L-Norleucine as an internal standard. $10 \mu \mathrm{l}$ from each sample were injected with an Agilent 1260 Infinity pump with a Nova-Pak C18 column and fluorescence detector, and Agilent Chemstation software for data recording. Amino acid derivatives were detected with an excitation wavelength of $250 \mathrm{~nm}$ and an emission wavelength of $395 \mathrm{~nm}$. Peak areas were compared to a standard curve made from a serial dilution of amino acid standards (Sigma-Aldrich, St. Louis, MO). injected into a Agilent 1260 Infinity HPLC (Agilent, Santa Clara, CA) with a Nova-Pak C18 column (Casteel et al., 2014). Solvent A, AccQ-Tag Eluent A, was premixed from Waters; Solvent B was acetonitrile:water (60:40). The gradient used was 0-0.01 min, $100 \% \mathrm{~A}$; 0.01-0.5 min, linear gradient to $3 \% \mathrm{~B}$; $0.5-12 \mathrm{~min}$, linear gradient to $5 \% \mathrm{~B} ; 12-15 \mathrm{~min}$, linear gradient to $8 \% \mathrm{~B} ; 15-45 \mathrm{~min}, 35 \% \mathrm{~B} ; 45-49 \mathrm{~min}$, linear gradient to $35 \% \mathrm{~B} ; 50-60 \mathrm{~min}, 100 \% \mathrm{~B}$. The flow rate was $1.0 \mathrm{ml} \mathrm{min}^{-1}$. Amino acid derivatives were measured with an Agilent fluorescence detector with an excitation wavelength of $250 \mathrm{~nm}$ and an emission wavelength of 395 nm. For concentration calculations, standard curves were generated for each amino acid using dilutions of the standard.

\subsection{Data analysis}

To evaluate effects of our treatments on host-plant defenses and host-plant quality, we ran a series of multivariate models using $\mathrm{R}$ ver. 3.5.2 (R Working Group, 2018). First, gene expression was evaluated withICS1 , LOX2 , GA2ox , AO3 , PR1 ,DRR230 , and PsLectin as the responses, with MANOVA to assess treatment effects on relative gene expression $\left(2^{-[?][?] \mathrm{Ct}}\right)$ based on cycle threshold values for each observed gene transcript. Estimated marginal mean of $\mathrm{Ct}$ values, and standard error of the mean, were generated using the emmeans package in $\mathrm{R}$ (Lenth, 2016). The methodology for $2^{-[?][?] \mathrm{Ct}}$ followed modified recommendations from Rao, Huang, Zhou, \& Lin (2013) and Kozera \& Rapacz (2013), using housekeeping gene $\beta$ - $\imath v \beta v \lambda \iota \nu$ to normalize expression and a sham aphid (non-infective Pea aphid and no weevil addition) treatment as a control.

Hormone levels in plants were evaluated using MANOVA, with salicylic acid, jasmonic acid, and abscisic acid as responses (3 variables). Total amino acid content was evaluated using a generalized linear model (GLM) with total concentration among all amino acids as the response. All models assessed treatment effects, using $S$. lineatus addition, $A$. pisum infection status, and their interaction as predictors. Finally, changes in the amino acid profile was evaluated using non-metric multidimensional scaling (NMDS) with the vegan package (Oksanen et al., 2019) following Ceulemans, Hulsmans, Ende, \& Honnay (2017).

\section{RESULTS}

3.1 Effects of multiple antagonists and attack order on plant gene transcripts 
Transcription of plant genes associated with hormone biosynthesis across four pathways: (i) salicylic acid (ICS1 ), (ii) jasmonic acid (LOX2), (iii) abscisic acid (AO3), and (iv) gibberellic acid (GA2ox), were induced by PEMV when $S$. lineatus was not present (Fig. 2A-D, Table S1, Pillai $=0.942, P=0.002$ ). However, there was no induction of any of these biosynthesis genes in response to PEMV when S. lineatus was present, indicating that $S$. lineatus inhibited plant defense against PEMV (Table S1, A x W interaction, Pillai $=1.509, P=0.021$, Fig. 2). In both the presence and absence of PEMV, S. lineatusinduced transcription of LOX2, but $S$. lineatus did not directly modify the expression of ICS1, AO3 or GA2ox (Fig. 2B-D, Table S1). When PEMV-infectious A. pisum attacked following S. lineatus, there was greater induction of LOX2 compared to when S. lineatus attacked alone (Table S1, A x W interaction, Pillai= $1.509, P=0.021$, Fig. 2). In contrast to the antagonism exerted by $S$. lineatus on the response to PEMV, this represents enhancement of plant defense when PEMV infection followed attack by $S$. lineatus .

All three defense response transcripts (PR1, DDR230,PsLectin) were induced by PEMV when S. lineatus was not present (Fig. 3); similarly, each transcript was induced by S. lineatus when PEMV was not present (Fig. 3, Table S1; A W interaction, $F=2.64, P=0.111$ ). When $S$. lineatusattacked second, the expression level of PR1 and Lectindid not change compared to when weevils were absent. The effects of PEMV on the transcripts was modified by the presence of $S$. lineatusand attack order. While DDR230 was induced by PEMV (Table S1, $F=47.181, P<0.001$ ), this effect diminished when $S$. lineatus was present after PEMV (Fig. 3B). Similarly, the effects of PEMV on PR1 were inhibited when S. lineatusattacked second (Fig. 3), whereas that the induction of lectin by PEMV was not altered by S. lineatus in either order (Fig. 3).

\subsection{Effects of multiple antagonists and attack order on plant phytohormones}

We observed variation in phytohormones in response to $A$. pisum(Table S2, Pillai $=0.95, P<0.001$ ) and $S$. lineatus (Table S2, Pillai $=1.195, \mathrm{P}<0.001$ ). PEMV-infectious $A$. pisum strongly induced salicylic acid (Table S2, $F=254.2, P<0.001$ ), but this was inhibited when $S$. lineatus attacked after PEMV (Fig. 4A, Tukey HSD). PEMV did not affect jasmonic acid (Table S2, $F=0.97, \mathrm{P}=0.34$ ), but the order of $S$. lineatus did (Table S2, $F=5.30, P=0.018$ ). Both $S$. lineatus (Table S2, $F=4.10, \mathrm{P}=0.037$ ) and infectious $A$. pisum induced abscisic acid (Table $\mathrm{S} 2, F=9.96, \mathrm{P}=0.006$ ) and this effect was contingent on the attack order (Table S2, A W, $F=4.32, P=0.032$, Fig. 4, Tukey HSD). Jasmonic acid levels were suppressed by S. lineatus when attacking prior to non-infectious sham A. pisum, but not on plants already attacked by PEMV (Fig. 4B, Tukey HSD).

\subsection{Effects of multiple antagonists and attack order on plant nutrients}

Feeding by $S$. lineatus increased the total amino acid levels (GLM, $\chi^{2}=9.19, P=0.01$, Fig 5), but PEMVinfectious $A$. pisum did not (GLM, $\chi^{2}=0.044, P=0.83$ ), and this effect was not modified depending on attack order (GLM, A W interaction, $\chi^{2}=0.24, P=0.63$ ). Non-metric multidimensional scaling (NMDS) analysis of amino acid composition also showed that changes to amino acid availability was most different among treatments for alanine, arginine, lysine, and glycine (Ordination plot, Fig S1).

\section{DISCUSSION}

Assessing interactions between biotic stressors and plants in food webs is critical to understand the dynamics of these interactions. Plant responses to stressors are often specific to the attacker, and both phytochemical responses and plant nutritional status can affect susceptibility to specific stressors (van Geem, Gols, Raaijmakers, \& Harvey, 2016; Shikano, 2017). We show plants responded in complex ways to unique biotic stressors, including a piercing-sucking herbivore (A. pisum ), a chewing herbivore (S. lineatus), and a virus (PEMV). Our study is among the first to assess how the order of attack, and diversity of stressors, mediate the defensive responses of plants and plant nutritional status (see also Vos, Moritz, Pieterse, \& Van Wees, 2015). Our results show that plants traits varied in response to the type of attacker, the number of stressors, and their order of arrival. Moreover, we show that assessment of multiple gene transcripts, phytohormones, and plant nutrients provides a more comprehensive perspective on mechanisms driving plant-insect-pathogen interactions than any isolated response. 
We found PEMV caused broad defensive responses in P. sativum by inducing specific gene transcripts and phytohormones (Figs. 2, $3 \& 4$ ). Biotropic pathogens such as PEMV are known to activate salicylic acid signaling (Singh, Swain, Singh, \& Nandi, 2018; Chisholm, Sertsuvalkul, Casteel, \& Crowder, 2018). This was reflected by increased expression of the ICS1 biosynthesis gene, increased salicylic acid hormone levels, and increased expression of the downstream defensive transcriptPR1 when PEMV was present. However, effects of PEMV were not limited to salicylic acid, as PEMV induced gene transcripts often associated with biosynthesis of abscisic acid (AO3), and giberrellic acid (GA20x), while also affecting defense response genes that occur downstream from induction of these hormones (PR1, DRR230 , PsLectin ). However, PEMV alone can not induce gene transcript associated with biosynthesis of JA ( $L O X 2$ ), but PEMV infection after weevil herbivory induced LOX2 accumulation. Similar results in P. sativum have been observed in response to fungal infection by Mycosphaerella pinodes and Phoma koolunga, where infections induce defense related genes across multiple signaling pathways (Fondevilla et al., 2011; Tran, You, \& Barbetti, 2018). However, increased expression of $L O X_{2}$ and $A O 3$ gene transcripts (Fig. 2) were not reflected by increased levels of jasmonic acid or abscisic acid (Fig. 4). This suggests that measuring phytohormones, or gene transcripts, in isolation may fail to reveal more complex pathways by which plants respond to stress (Kazan \& Lyons, 2014).

While PEMV had broad effects on plants, S. lineatus attenuated these responses. When S. lineatus was present, before or after PEMV, the expression of three out of four biosynthesis gene transcripts (with the exception of LOX2) in response to infectious aphids were comparatively weaker (Fig. 2). Increased expression of $L O X 2$ may be due to $S$. lineatus inducing expression of defensive transcripts associated with JA-mediated chewing herbivore attack and the effect was further enhanced by PEMV infection after $S$. lineatusfeeding (Fig. 2). Effects of PEMV on plant defense genes (PR1,DDR230, PsLectin ) were also affected by $S$. lineatus, but varied with attack order. Overall, in this study the order of attack seemed to have stronger effects on downstream plant defense gene transcripts than on hormone biosynthesis gene transcripts.

While $S$. lineatus increased expression of two (LOX2 andGA2ox) of the four biosynthesis gene transcripts studied when PEMV was not present, expression of $L O X 2$ was enhanced when PEMV was also present (Fig. 2). In contrast, PEMV caused decreased expression of two genes $(P R 1, D R R 230)$ that were induced by $S$. lineatus was present alone (Fig. 2). For plants attacked first by either PEMV or S. lineatus, we observed the strongest evidence for mutual antagonism at the gene transcript level rather than for phytohormones (Figs. 2-4). Our study shows interactions among a set of stressors can vary based on attack order. Here, we found that $S$. lineatus feeding following PEMV infection inhibited plant defense (mutual antagonism), while LOX2 expression was enhanced when PEMV infection followed S. lineatus feeding (synergistic effect). While the first effect is in line with studies showing "mutual antagonism" between chewing herbivores and biotropic pathogens (Thaler, Agrawal, \& Halitschke, 2010; Vos et al., 2015), our study suggests the order of attack can lead to variation along a spectrum from antagonism to enhancement.

Our results provide evidence that the order of arrival of biotic stressors on plants can play a crucial role in determining plants' response to these attackers. While mutual antagonism between $S$. lineatus and PEMV was common, for some genes these effects only occurred when $S$. lineatus attacked first, and for others when $S$. lineatus attacked second (Figs. 2-4). Mutual antagonism has most often been studied as effects of a prior attacker affecting a subsequent attacker, such as when a herbivore alters gene activation or phytohormones in ways that attenuate performance of subsequent attackers (Kessler \& Halitschke, 2007; Erb, Robert, Hibbard, \& Turlings, 2011; Stam, Mantelin, McLellan, \& Thilliez, 2014; Huang et al., 2017). However, our results suggest that a second attacker may also mitigate defensive responses against the first attacker in ways that might affect plant defense and propagation of pathogens. For example, we show that plants infected by PEMV had decreased defenses when subsequently attacked by S. lineatus (Fig. 3), which should promote PEMV replication. Moreover, our results suggest that, PEMV infection induces pathogen defense and $S$. lineatus inhibits that if they appear on plants after the infection has been established. This may be more strongly expressed as variation in gene transcripts rather than hormone levels, a result that has similarly been seen in Arabidopsis in response to pathogen infection (Anderson et al., 2004). 
Mutual antagonism in plant signaling pathways has most commonly been examined in regard to tradeoffs between jasmonic acid and salicylic acid. Our results show these tradeoffs extend to other signaling pathways. For example, jasmonic acid exhibits antagonism with abscisic acid in Arabidopsis following attack from Fusarium oxysporum (Anderson et al., 2004). Mutual antagonism between jasmonic acid and gibberellic acid, and jasmonic acid and abscisic acid, have also been reported (Yang, Yang, \& He, 2013; Okada et al., 2015; Liu \& Hou, 2018). For example, jasmonic acid facilitates defense over growth by repressing degradation of DELLA protein in rice andArabidopsis, but elevated DELLA proteins interfere with the gibberellic acid pathway by binding to growth promoting transcription factors associated with gibberellic acid signaling (Yang et al., 2012, 2013; Okada et al., 2015). Antagonistic relationships between giberellic acid and abscisic acid have also been reported in both mono and dicot plants and regulated by various transcription factor regulators in response to diverse environmental cues (Liu \& Hou, 2018). However, antagonisms between salicylic acid and abscisic acid may actually lead to synergism between jasmonic acid and abscisic acid, where elevated abscisic acid levels following infection with Pseudomonas syringae induce jasmonic acid in Arabidopsis, which in turn limits the levels of salicylic acid (Fan, Hill, Crooks, Doerner, \& Lamb, 2009). Overall, these results suggest that a broad examination of genes and hormones are needed to elucidate pathways underlying plant-insect-pathogen interactions in $P$. sativum and other plants.

Our results suggest mutual antagonism may also occur among defense gene transcripts that are associated with a single signaling pathway. For example, the induction of $P R 1$, a salicylic acid-responsive gene, was mitigated by $S$. lineatus attack after PEMV infection, as may be expected with antagonism between jasmonic acid and salicylic acid. However, the expression of ICS1, another gene associated with the biosynthesis of salicylic acid, was not responsive to $S$. lineatus . This has been seen in other studies where ICS1 was not induced by caterpillar feeding although other genes associated with salicylic acid were (Onkokesung, Reichelt, van Doorn, Schuurink, \& Dicke 2016). These results suggest that a plant's response to multiple stressors is unlikely to result from simple crosstalk but rather from interactions among multiple signaling pathways that may exhibit complex responses.

In addition to affecting plant gene expression and phytohormones, plant pathogens such as viruses can also alter nutritional quality of their host plants in ways that affect vectors (Mauck, Bosque-Pérez, Eigenbrode, Moraes, \& Mescher, 2012; Wang, Senthil-Kumar, Ryu, Kang, \& Mysore, 2012; Patton et al., 2019). Similarly, non-vector herbivores may strongly affect the quantity and quality of plant nutrients (Ángeles-López, RiveraBustamante, \& Heil, 2016). For example,pepper golden mosaic virus (PGMV) infection in Capsicum annuum increased levels of the amino acids proline, tyrosine, valine but decreased levels of histidine and alanine. In the same system, the greenhouse whitefly, Trialeurodes vaporarioum, reversed the levels of these amino acids (Ángeles-López et al., 2016). Arrival of S. lineatus before PEMV infection suppressed the amount of total amino acids in peas, while enhanced amino acid level was detected if $S$. lineatus damaged peas after PEMV infected was established. This suggests the intriguing possibility that antagonism between a pathogen and non-vector herbivore can occur at the level of amino acid production in plants.

Overall, our study provides example of complex interactions between a vector-borne plant pathogen and a non-vector herbivore that varies from antagonism to enhancement and manifest as changes in plant gene transcripts, phytohormones levels, and plant nutrients. However, we show that assessing the order of attack is necessary to best understand the complexity and mechanisms of plant-insect-pathogen interactions. Moreover, our study suggests complete pathways must be characterized as differences are evident even when a few transcripts and metabolites are analyzed., often measured with associated gene transcripts (Bedini, Mercy, Schneider, Franken, \& Lucic-Mercy, 2018; Ángeles-López el al., 2016; Shi et al., 2019), may fail to capture mechanisms by which plants interact with multiple stressors. Our results demonstrate both the order of arrival, and the diversity of interactions, determine plant responses to stress through the combined action of defense gene activation, phytohormone accumulation, and modification of plant nutrients. Characterizing the pathways by which plants respond to single and multiple stressors, with varying attack order, can shed light on the mechanisms that shape food web interactions among plants, herbivores, and pathogens.

\section{ACKNOWLEDGEMENTS}


We thank the many undergraduates who helped in various experiments, data collection, and O. Hill for assistance with data analysis. This research was supported by USDA-NIFA Grants 2016-67011-24693, 201767013-26537, and Hatch project 1014754.

\section{REFERENCES}

Anderson, J. P., Badruzsaufari, E., Schenk, P. M., Manners, J. M., Desmond, O. J., Ehlert, C., .. Kazan, K. (2004). Antagonistic interaction between abscisic acid and jasmonate-ethylene signaling pathways modulates defense gene expression and disease resistance inArabidopsis . The Plant Cell , 16 (12), 3460-3479. doi: $10.1105 /$ tpc. 104.025833

Angeles-Lopez, Y. I., Rivera-Bustamante, R. F., \& Heil, M. (2016). Colonization by phloem-feeding herbivore overrides effects of plant virus on amino acid composition in phloem of chili plants. Journal of Chemical Ecology , 42 (10), 985-988. doi: 10.1007/s10886-016-0747-2

Armijo, G., Salinas, P., Monteoliva, M. I., Seguel, A., Garcia, C., Villarroel-Candia, E., .. Holuigue, L. (2013). A salicylic acid-induced lectin-like protein plays a positive role in the effector-triggered immunity response of Arabidopsis thaliana toPseudomonas syringae Avr-Rpm1. Molecular Plant-Microbe Interactions: MPMI , 26 (12), 1395-1406. doi: 10.1094/MPMI-02-13-0044-R

Bedini, A., Mercy, L., Schneider, C., Franken, P., \& Lucic-Mercy, E. (2018). Unraveling the initial plant hormone signaling, metabolic mechanisms and plant defense triggering the endomycorrhizal symbiosis behavior. Frontiers in Plant Science, 9 . doi: 10.3389/fpls.2018.01800

Behmer, S. T. (2009). Insect herbivore nutrient regulation. Annual Review of Entomology, 54 (1), 165-187. doi: 10.1146/annurev.ento.54.110807.090537

Bera, S., Blundell, R., Liang, D., Crowder, D. W., \& Casteel, C. L. (2020). The oxylipin signaling pathway is required for increased aphid attraction and retention on virus-infected plants. Journal of Chemical Ecology . doi: 10.1007/s10886-020-01157-7

Black, A. E., Strand, E., Wright, R. G., Scott, J. M., Morgan, P., \& Watson, C. (1998). Land use history at multiple scales: implications for conservation planning. Landscape and Urban Planning ,43 (1), 49-63. doi: 10.1016/S0169-2046(98)00096-6

Carcamo, H. A., Vankosky, M. A., Olfert, O. O., Wijerathna, A., Evenden, M. L., \& Meers, S.B. (2018). Progress toward integrated pest management of pea leaf weevil: a review. Annals of the Entomological Society of America , 111(4), 144-153. (doi:10.1093/aesa/say007)

Casteel, C. L., Yang, C., Nanduri, A. C., De Jong, H. N., Whitham, S. A., \& Jander G. (2014). The NIa-Pro protein of Turnip mosaic virus improves growth and reproduction of the aphid vector, Myzus persicae (green peach aphid). Plant Journal , 77, 653-663. doi:

\subsection{1/tpj.12417}

Ceulemans, T., E. Hulsmans, W. V. Ende, \& O. Honnay. (2017). Nutrient enrichment is associated with altered nectar and pollen chemical composition in Succisa pratensis (Moench) and increased larval mortality of its pollinator Bombus terrestris L. PLOS ONE12 (4), e0175160. doi: 10.1371/journal.pone.0175160

Chiozza, M. V., O’Neal, M. E., \& MacIntosh, G. C. (2010). Constitutive and induced differential accumulation of amino acid in leaves of susceptible and resistant soybean plants in response to the soybean aphid (Hemiptera: Aphididae). Environmental Entomology ,39 (3), 856-864. doi: 10.1603/EN09338

Chisholm, P. J., Eigenbrode, S. D., Clark, R. E., Basu, S., \& Crowder, D. W. (2019). Plant-mediated interactions between a vector and a non-vector herbivore promote the spread of a plant virus.Proceedings of the Royal Society B: Biological Sciences ,286 (1911), 20191383. doi: 10.1098/rspb.2019.1383

Chisholm, P. J., Sertsuvalkul, N., Casteel, C. L., \& Crowder, D. W. (2018). Reciprocal plant-mediated interactions between a virus and a non-vector herbivore. Ecology, 99 (10), 2139-2144. doi: 10.1002/ecy.2449 
Cipollini, D., Walters, D., \& Voelckel, C. (2017). Costs of resistance in plants: from theory to evidence. In Annual Plant Reviews online (pp. 263-307). doi: 10.1002/9781119312994.apr0512

Erb, M., Robert, C. A. M., Hibbard, B. E., \& Turlings, T. C. J. (2011). Sequence of arrival determines plant-mediated interactions between herbivores. Journal of Ecology , 99 (1), 7-15. doi: 10.1111/j.13652745.2010.01757.x

Fan, J., Hill, L., Crooks, C., Doerner, P., \& Lamb, C. (2009). Abscisic acid has a key role in modulating diverse plant-pathogen interactions.Plant Physiology , 150 (4), 1750-1761. doi: 10.1104/pp.109.137943

Fondevilla, S., Kuster, H., Krajinski, F., Cubero, J. I., \& Rubiales, D. (2011). Identification of genes differentially expressed in a resistant reaction to Mycosphaerella pinodes in pea using microarray technology. BMC Genomics , 12 , 28. doi: 10.1186/1471-2164-12-28

Guo, L., Su, Q., Yin, J., Yang, Z., Xie, W., Wang, S., .. Zhang, Y. (2019). Amino acid utilization may explain why Bemisia tabaci $\mathrm{Q}$ and $\mathrm{B}$ differ in their performance on plants infected by the Tomato yellow leaf curl virus . Frontiers in Physiology ,10 . doi: 10.3389/fphys.2019.00489

He, H., Liang, G., Lu, S., Wang, P., Liu, T., Ma, Z., Zuo, C., Sun, X., Chen, B., \& Mao, J. (2019). Genomewide identification and expression analysis of GA2ox, GA3ox, and GA20ox are related to gibberellin oxidase genes in grape (VitisVinifera L.). Genes, 10 (9), 680. https://doi.org/10.3390/genes10090680

Huang, W., Robert, C. A. M., Herve, M. R., Hu, L., Bont, Z., \& Erb, M. (2017). A mechanism for sequence specificity in plant-mediated interactions between herbivores. New Phytologist , 214 (1), 169-179. doi: 10.1111/nph.14328

Kazan, K., \& Lyons, R. (2014). Intervention of phytohormone pathways by pathogen effectors. The Plant Cell , 26 (6), 2285-2309. doi: 10.1105/tpc.114.125419

Kessler, A., \& Halitschke, R. (2007). Specificity and complexity: the impact of herbivore-induced plant responses on arthropod community structure. Current Opinion in Plant Biology , 10 (4), 409-414. doi: 10.1016/j.pbi.2007.06.001

Koornneef, A., \& Pieterse, C. M. J. (2008). Cross talk in defense signaling. Plant Physiology , 146 (3), 839-844. doi: 10.1104/pp.107.112029

Kozera, B., \& Rapacz, M. (2013). Reference genes in real-time PCR.Journal of Applied Genetics , 54 (4), 391-406. doi: 10.1007/s13353-013-0173-x

Kreplak, J., Madoui, M.-A., Capal, P., Novak, P., Labadie, K., Aubert, G., .. Burstin, J. (2019). A reference genome for pea provides insight into legume genome evolution. Nature Genetics ,51 (9), 14111422. doi: $10.1038 / \mathrm{s} 41588-019-0480-1$

Kulaeva, O. A., Zhernakov, A. I., Afonin, A. M., Boikov, S. S., Sulima, A. S., Tikhonovich, I. A., \& Zhukov, V. A. (2017). Pea Marker Database (PMD) - A new online database combining known pea (Pisum sativumL.) gene-based markers. PLOS ONE , 12 (10), e0186713. doi: 10.1371/journal.pone.0186713

Lacerda, A. F., Vasconcelos, E. A. R., Pelegrini, P. B., \& Grossi de Sa, M. F. (2014). Antifungal defensins and their role in plant defense.Frontiers in Microbiology , 5 , 116. doi: 10.3389/fmicb.2014.00116

Lee, Y., Kim, Y.-C., Kim, S. Y., Lee, I.-J., Choi, D., Paek, K.-H., .. Park, J. M. (2012). A novel gibberellin 2-oxidase geneCaGA2ox1 in pepper is specifically induced by incompatible plant pathogens. Plant Biotechnology Reports , 6 (4), 381-390. doi: 10.1007/s11816-012-0235-2

Lenth, R.V. 2016. Least-Squares Means: The R package lsmeans. Journal of Statistical Software, 69:1-33.

Lin, D., Xu, Y., Wu, H., Liu, X., Zhang, L., Wang, J., \& Rao, Q. (2019). Plant defense responses induced by two herbivores and consequences for whitefly Bemisia tabaci . Frontiers in Physiology , 10 . doi: 10.3389/fphys.2019.00346 
Liu, X., \& Hou, X. (2018). Antagonistic regulation of ABA and GA in metabolism and signaling pathways. Frontiers in Plant Science, 9 . doi: 10.3389/fpls.2018.00251

Livak, K. J., \& Schmittgen, T. D. (2001). Analysis of relative gene expression data using real-time quantitative PCR and the 2(-Delta Delta C(T)) Method. Methods (San Diego, Calif.) , 25 (4), 402-408. doi: 10.1006/meth.2001.1262

Macedo, M. L. R., Oliveira, C. F. R., \& Oliveira, C. T. (2015). Insecticidal activity of plant lectins and potential application in crop protection. Molecules , 20 (2), 2014-2033. doi: 10.3390/molecules20022014

Mauch-Mani, B., Baccelli, I., Luna, E., \& Flors, V. (2017). Defense priming: an adaptive part of induced resistance. Annual Review of Plant Biology , 68 (1), 485-512. doi: 10.1146/annurev-arplant-042916-041132

Mauck, K., Bosque-Perez, N. A., Eigenbrode, S. D., Moraes, C. M. D., \& Mescher, M. C. (2012). Transmission mechanisms shape pathogen effects on host-vector interactions: evidence from plant viruses. Functional Ecology , 26 (5), 1162-1175. doi: 10.1111/j.1365-2435.2012.02026.x

Miller, R. N. G., Costa Alves, G. S., \& Van Sluys, M.-A. (2017). Plant immunity: unravelling the complexity of plant responses to biotic stresses. Annals of Botany , 119 (5), 681-687. doi: 10.1093/aob/mcw284

Miranda, V. de J., Porto, W. F., Fernandes, G. da R., Pogue, R., Nolasco, D. O., Araujo, A. C. G., ... Franco, O. L. (2017). Comparative transcriptomic analysis indicates genes associated with local and systemic resistance to Colletotrichum graminicola in maize. Scientific Reports , 7 (1), 2483. doi: 10.1038/s41598017-02298-8

Mur, L. A. J., Simpson, C., Kumari, A., Gupta, A. K., \& Gupta, K. J. (2017). Moving nitrogen to the centre of plant defence against pathogens. Annals of Botany , 119 (5), 703-709. doi: 10.1093/aob/mcw179

Nejat, N., \& Mantri, N. (2017). Plant immune system: crosstalk between responses to biotic and abiotic stresses the missing link in understanding plant defence. Current Issues in Molecular Biology ,23 , 1-16. doi: $10.21775 /$ cimb.023.001

Okada, K., Abe, H., \& Arimura, G. (2015). Jasmonates induce both defense responses and communication in monocotyledonous and dicotyledonous plants. Plant $\&$ Cell Physiology , 56 (1), 16-27. doi: $10.1093 / \mathrm{pcp} / \mathrm{pcu} 158$

Oksanen, F.J. Guillaume Blanchet, Michael Friendly, Roeland Kindt, Pierre Legendre, Dan McGlinn, Peter R. Minchin, R. B. O'Hara, Gavin L. Simpson, Peter Solymos, M. Henry H. Stevens, Eduard Szoecs and Helene Wagner (2019). vegan: Community ecology package. R package version 2.5-5. https://CRAN.Rproject.org $/$ package $=$ vegan

Onkokesung, N., Reichelt, M., van Doorn, A., Schuurink, R. C., \& Dicke, M. (2016). Differential Costs of Two Distinct Resistance Mechanisms Induced by Different Herbivore Species in Arabidopsis. Plant physiology, 170(2), 891-906. doi:10.1104/pp.15.01780

Pandey, P., Ramegowda, V., \& Senthil-Kumar, M. (2015). Shared and unique responses of plants to multiple individual stresses and stress combinations: physiological and molecular mechanisms. Frontiers in Plant Science, 6 . doi: 10.3389/fpls.2015.00723

Patton, M. F., Bak, A., Sayre, J. M., Heck, M. L., \& Casteel, C. L. (2020). A polerovirus, Potato leafroll virus, alters plant-vector interactions using three viral proteins. Plant, Cell 83 Environment, 43 (2), 387-399. doi: 10.1111/pce.13684

Petek, M., Rotter, A., Kogovšek, P., Baebler, S., Mithöfer, A., \& Gruden, K. (2014). Potato virus Y infection hinders potato defence response and renders plants more vulnerable to Colorado potato beetle attack. Molecular ecology , 23(21), 5378-5391. doi: org/10.1111/mec.12932

Ramírez-Carrasco, G., Martínez-Aguilar, K., \& Alvarez-Venegas, R. (2017). Transgenerational defense priming for crop protection against plant pathogens: A hypothesis. Frontiers in Plant Science ,8 . doi: 
10.3389/fpls.2017.00696

Rao, X., X. Huang, Z. Zhou, and X. Lin. 2013. An improvement of the $2^{(}-$ -deltadeltaCT)methodforquantitativereal-timepolymerasechainreactiondataanalysis.Biostatistics, bioinformatics and $b$ -85 .

Seguel, A., Jelenska, J., Herrera-Vásquez, A., Marr, S. K., Joyce, M. B., Gagesch, K. R., ... Holuigue, L. (2018). PROHIBITIN3 forms complexes with ISOCHORISMATE SYNTHASE1 to regulate stress-induced salicylic acid biosynthesis in Arabidopsis . Plant Physiology , 176 (3), 2515-2531. doi: 10.1104/pp.17.00941

Selim, S., Sanssené, J., Rossard, S., \& Courtois, J. (2017). Systemic induction of the defensin and phytoalexin pisatin pathways in pea (Pisum sativum) against Aphanomyces euteiches by acetylated and nonacetylated oligogalacturonides. Molecules ,22 (6), 1017. doi: 10.3390/molecules22061017

Serova, T. A., Tsyganova, A. V., Tikhonovich, I. A., \& Tsyganov, V. E. (2019). Gibberellins inhibit nodule senescence and stimulate nodule meristem bifurcation in pea (Pisum sativum L.). Frontiers in Plant Science , 10 . doi: $10.3389 /$ fpls.2019.00285

Shi, J.-H., Sun, Z., Hu, X.-J., Jin, H., Foba, C. N., Liu, H., .. Wang, M.-Q. (2019). Rice defense responses are induced upon leaf rolling by an insect herbivore. BMC Plant Biology , 19 (1), 514. doi: 10.1186/s12870019-2116-0

Shikano, I. (2017). Evolutionary ecology of multitrophic interactions between plants, insect herbivores and entomopathogens. Journal of Chemical Ecology , 43 (6), 586-598. doi: 10.1007/s10886-017-0850-z

Singh, N., Swain, S., Singh, A., \& Nandi, A. K. (2018). AtOZF1 positively regulates defense against bacterial pathogens and npr1-independent salicylic acid signaling. Molecular Plant-Microbe Interactions , 31 (3), 323333. doi: 10.1094/MPMI-08-17-0208-R

Stam, R., Mantelin, S., McLellan, H., \& Thilliez, G. (2014). The role of effectors in nonhost resistance to filamentous plant pathogens.Frontiers in Plant Science, 5 . doi: 10.3389/fpls.2014.00582

Su, Q., Mescher, M.C., Wang, S., Chen, G., Xie, W., Wu, Q., Wang, W., \& Zhang, Y. Tomato yellow leaf curl virus differentially influences plant defence responses to a vector and a non-vector herbivore. (2016).Plant, Cell \& Environment, 39(3), 597-607. doi:

$10.1111 /$ pce.12650

Suzuki, N. (2016). Hormone signaling pathways under stress combinations.Plant Signaling \& Behavior , 11 (11), e1247139. doi: 10.1080/15592324.2016.1247139

Thaler, J. S., Agrawal, A. A., \& Halitschke, R. (2010). Salicylate-mediated interactions between pathogens and herbivores.Ecology , 91 (4), 1075-1082. doi: 10.1890/08-2347.1

Thaler, J. S., Humphrey, P. T., \& Whiteman, N. K. (2012). Evolution of jasmonate and salicylate signal crosstalk. Trends in Plant Science, 17 (5), 260-270. doi: 10.1016/j.tplants.2012.02.010

Tran, H. S., You, M. P., \& Barbetti, M. J. (2018). Expression of defence-related genes in stems and leaves of resistant and susceptible field pea (Pisum sativum) during infection by Phoma koolunga. Plant Pathology , 67 (1), 156-166. doi: 10.1111/ppa.12709

van Geem, M., Gols, R., Raaijmakers, C. E., \& Harvey, J. A. (2016). Effects of population-related variation in plant primary and secondary metabolites on aboveground and belowground multitrophic interactions. Chemoecology , 26 (6), 219-233. doi: 10.1007/s00049-016-0222-0

Vos, I. A., Moritz, L., Pieterse, C. M. J., \& Van Wees, S. C. M. (2015). Impact of hormonal crosstalk on plant resistance and fitness under multi-attacker conditions. Frontiers in Plant Science ,6 . doi: 10.3389/fpls.2015.00639 
Wang, K., Senthil-Kumar, M., Ryu, C.-M., Kang, L., \& Mysore, K. S. (2012). Phytosterols play a key role in plant innate immunity against bacterial pathogens by regulating nutrient efflux into the apoplast.Plant Physiology , 158 (4), 1789-1802. doi: 10.1104/pp.111.189217

War, Abdul Rashid, Paulraj, M. G., Ahmad, T., Buhroo, A. A., Hussain, B., Ignacimuthu, S., \& Sharma, H. C. (2012). Mechanisms of plant defense against insect herbivores. Plant Signaling \&3 Behavior ,7 (10), 1306-1320. doi: 10.4161/psb.21663

Wasternack, C., \& Hause, B. (2013). Jasmonates: biosynthesis, perception, signal transduction and action in plant stress response, growth and development. An update to the 2007 review in Annals of Botany. Annals of Botany , 111 (6), 1021-1058. doi: 10.1093/aob/mct067

Yang, D.-L., Yang, Y., \& He, Z. (2013). Roles of plant hormones and their interplay in rice immunity. Molecular Plant , 6 (3), 675-685. doi: 10.1093/mp/sst056

Yang, D.-L., Yao, J., Mei, C.-S., Tong, X.-H., Zeng, L.-J., Li, Q., .. He, S. Y. (2012). Plant hormone jasmonate prioritizes defense over growth by interfering with gibberellin signaling cascade.Proceedings of the National Academy of Sciences of the United States of America , 109 (19), E1192-1200. doi: 10.1073/pnas.1201616109

Yergaliyev, T. M., Nurbekova, Z., Mukiyanova, G., Akbassova, A., Sutula, M., Zhangazin, S., .. Omarov, R. T. (2016). The involvement of ROS producing aldehyde oxidase in plant response to Tombusvirus infection.Plant Physiology and Biochemistry: PPB , 109 , 36-44. doi: 10.1016/j.plaphy.2016.09.001

Zdunek-Zastocka, E., \& Sobczak, M. (2013). Expression of Pisum sativum PsAO3 gene, which encodes an aldehyde oxidase utilizing abscisic aldehyde, is induced under progressively but not rapidly imposed drought stress. Plant Physiology and Biochemistry: PPB ,71 , 57-66. doi: 10.1016/j.plaphy.2013.06.027

Zhou, S., Lou, Y.-R., Tzin, V., \& Jander, G. (2015). Alteration of plant primary metabolism in response to insect herbivory. Plant Physiology , 169 (3), 1488-1498. doi: 10.1104/pp.15.01405

Zhu, F., Poelman, E. H., \& Dicke, M. (2014). Insect herbivore-associated organisms affect plant responses to herbivory.New Phytologist , 204 (2), 315-321. doi: 10.1111/nph.12886

\section{DATA ACCESSIBILITY}

Data are publicly available from Figshare: https://doi.org/10.6084/m9.figshare.14227097.v1

\section{AUTHOR CONTRIBUTIONS}

S.B. and D.W.C. conceived the ideas and designed the methodology; S.B., R.E.C., S.B. and C.L.C. collected the data; R.E.C., S.B., C.L.C and D.W.C. analyzed the data; all authors contributed critically to the drafts and gave final approval for publication.

\section{Figure legends}

Figure 1. Schematic representation of $2 \times 3$ factorial design. Green-colored aphids indicate sham (noninfective) A. pisum, while blue-colored aphids indicate PEMV-infective A. pisum. Slashes indicate order of $S$. lineatus treatments (S. lineatus first, A. pisum first, or no S. lineatus ).

Figure 2. Relative transcript accumulation of plant hormone biosynthesis genes associated with four hormonal signaling pathways: (A)ICS1 (salicylic acid), (B) LOX2 (jasmonic acid), (C)AOX3 (abscisic acid), and (D) GA2ox (gibberellic acid) following attack with various combinations of S. lineatus ,A. pisum, and PEMV. Within each panel, bars separated by a different letter were significant different based on MANOVA (Tukey HSD, $\alpha=0.05$ ). Bar height and error bars indicate marginal mean and standard error of the regression coefficient for each respective treatment.

Figure 3. Relative transcript accumulation of plant defense response transcripts: (A) PR1, (B) DDR230, and (C)PsLectin following attack with various combinations of S. lineatus, A. pisum, and PEMV. Within each panel, bars separated by a different letter were significant different based on MANOVA (Tukey HSD, $\alpha$ 
$=0.05)$. Bar height and error bars indicate marginal mean and standard error of the regression coefficient for each respective treatment.

Figure 4 . (A) Salicylic acid, (B) jasmonic acid, and (C) abscisic acid phytohormone levels in P. sativum plants following attack with various combinations of S. lineatus, A. pisum, and PEMV. Within each panel, bars not connected by the same letter are significantly different (Tukey HSD, $\alpha=0.05$ ). Bar height and error bars indicate marginal mean and standard error of the regression coefficient for each respective treatment.

Figure 5 . Nutritional analysis (total amino acid) in P. sativum following attack with various combinations of $S$. lineatus , A. pisum, and PEMV. S. lineatus increase total amino acid concentration in plants (GLM, $\chi 2=9.194, P=0.01)$. There was no "sham-none" treatment combination so that could not be estimated. Within each panel, bars not connected by the same letter are significantly different (Tukey HSD, $\alpha=0.05$ ). Bar height and error bars indicate marginal mean and standard error of the regression coefficient for each respective treatment.

Fig. 1

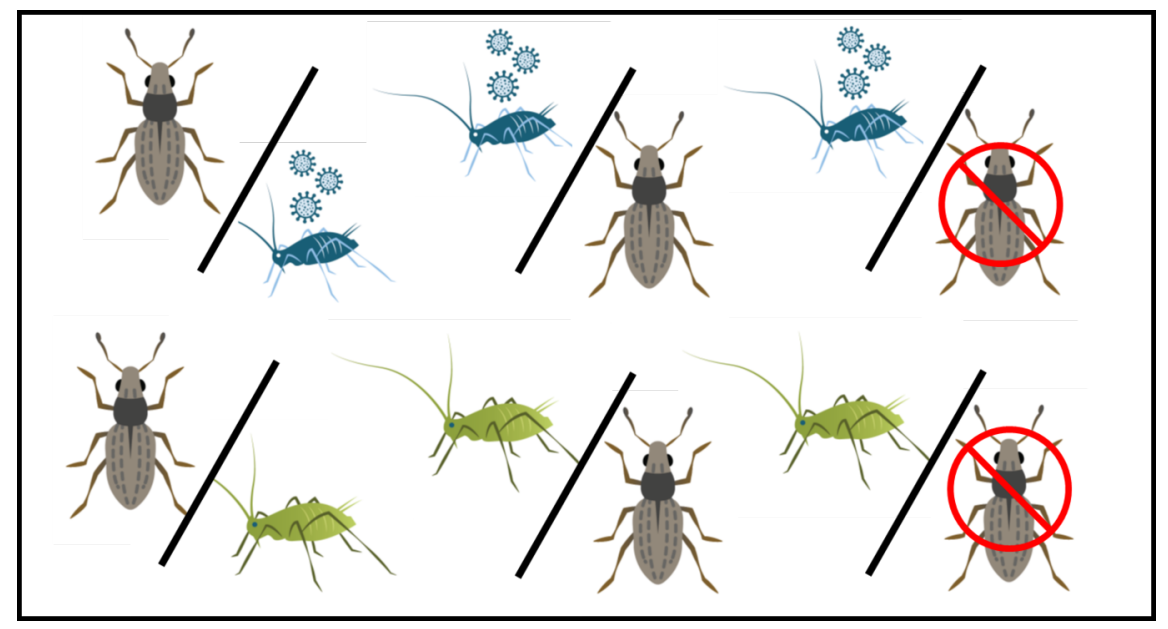

Fig. 2 


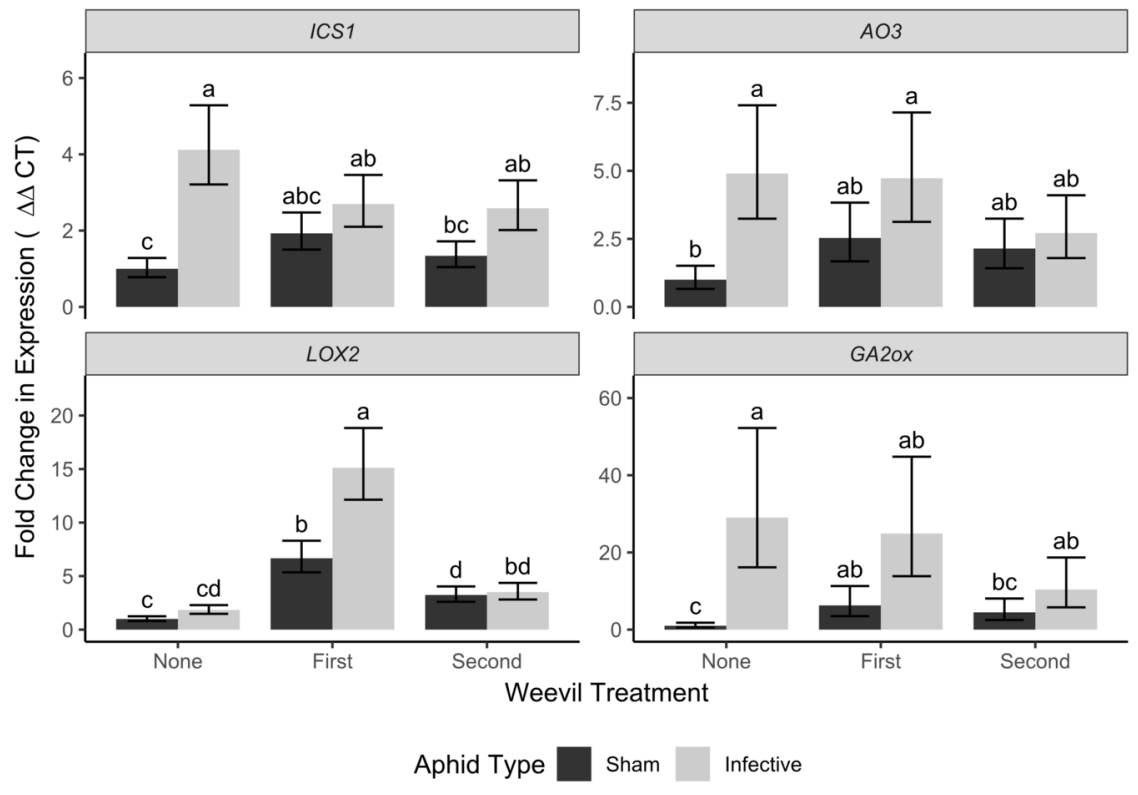

Fig. 3
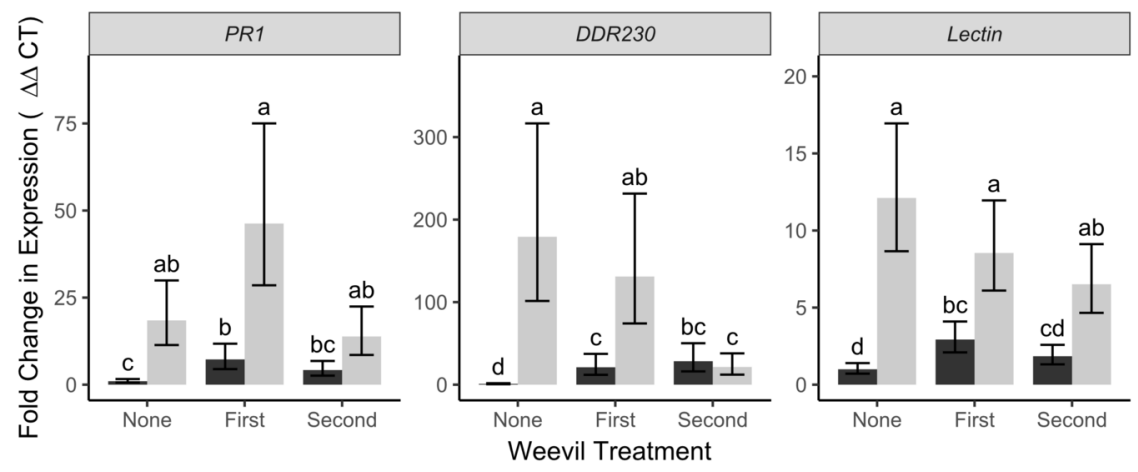

Aphid Type Sham Infective

Fig. 4 


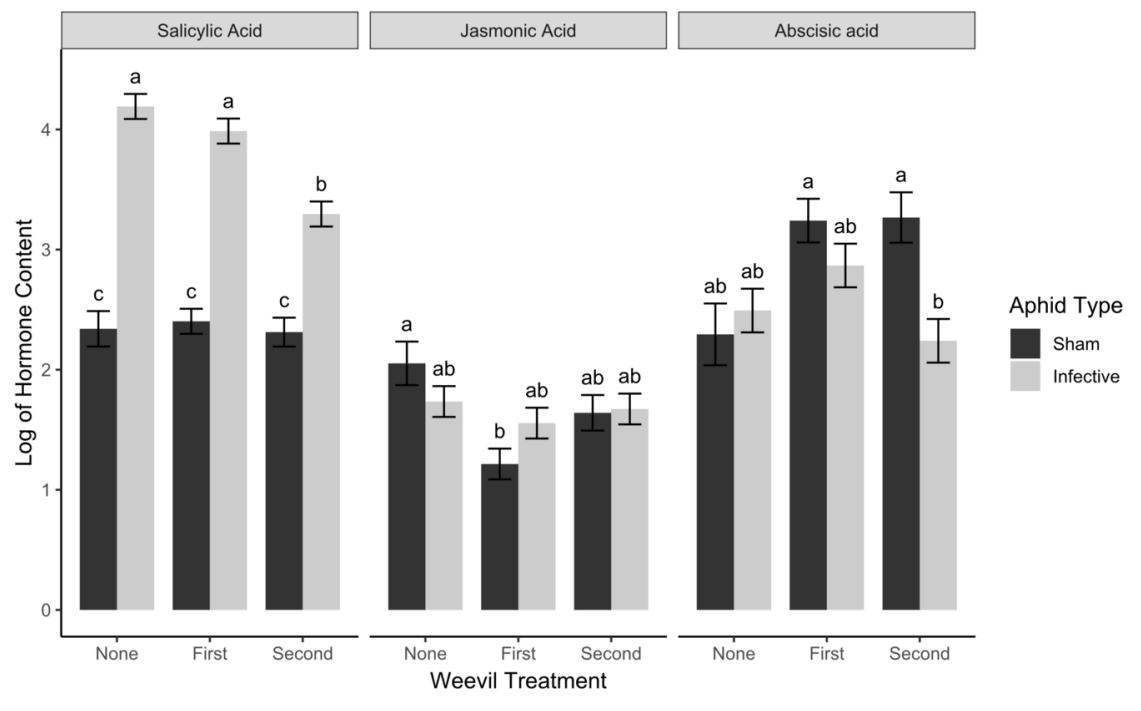

Fig. 5

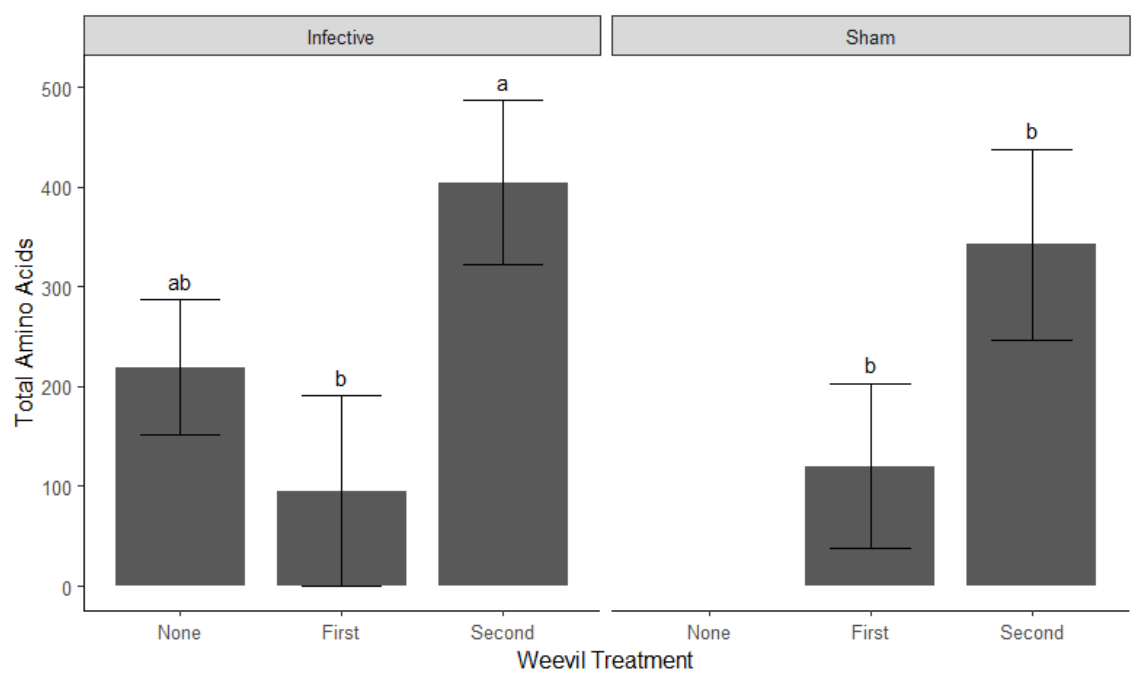

